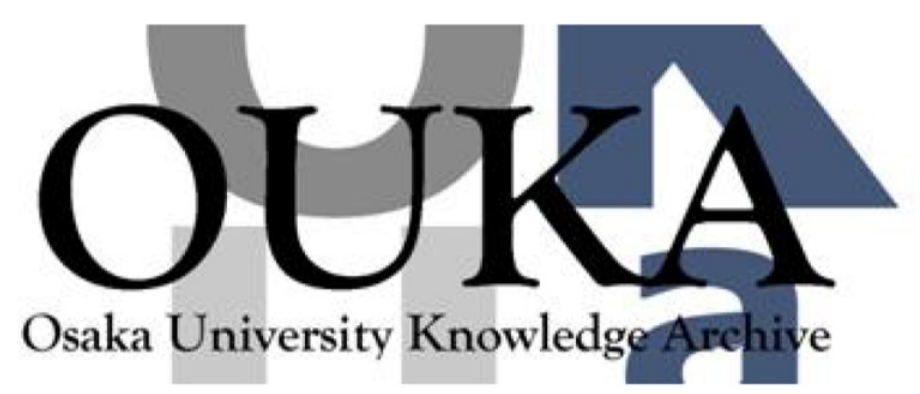

\begin{tabular}{|c|c|}
\hline Title & $\begin{array}{l}\text { Optical property of photonic crystals } \\
\text { infiltrated with various liquids and liquid } \\
\text { crystals }\end{array}$ \\
\hline Author (s) & Yoshino, K.; Ootake, R.; Takeda, M. et al. \\
\hline Citation & $\begin{array}{l}\text { IEEE International Conference on Conduction and } \\
\text { Breakdown in Dielectric Liquids, ICDL. p.393- } \\
\text { p. } 396\end{array}$ \\
\hline Issue Date & $2002-07$ \\
\hline oaire:version & VoR \\
\hline URL & https://hdl. handle. net/11094/14080 \\
\hline rights & $\begin{array}{l}\text { c2002 IEEE. Personal use of this material is } \\
\text { permitted. However, permission to } \\
\text { reprint/republish this material for advertising } \\
\text { or promotional purposes or for creating new } \\
\text { collective works for resale or redistribution } \\
\text { to servers or lists, or to reuse any } \\
\text { copyrighted component of this work in other } \\
\text { works must be obtained from the IEEE. }\end{array}$ \\
\hline Note & \\
\hline
\end{tabular}

Osaka University Knowledge Archive : OUKA

https://ir. Library. osaka-u. ac. jp/

Osaka University 


\title{
Optical Property of Photonic Crystals Infiltrated with Various Liquids and Liquid Crystals
}

\author{
K.Yoshino, R.Ootake, M.Takeda, A.Fujii and M.Ozaki \\ Department of Electronic Engineering,.Faculty of Engineering, Osaka University \\ Yamada-Oka 2-1, Suita, Osaka 55-0871 Japan
}

\begin{abstract}
Upon infiltration of various liquids in the opal and its polymer replicas as photonic crystal with a three-dimensional periodic structure of optical wavelength order, the color changed remarkably and a sharp reflection peak and an absorption dip change drastically. in wavelength. These shifts are strongly dependent on the sort of infiltrated liquids and can be explained by the difference of refractive index of the liquid. On the contrary from these spectral shifts refractive index of the infiltrated liquid is easily evaluated. Utilizing these spectral shift the refractive index of the liquid and in the case of mixed liquid the concentration of each component of liquid can be evaluated. Theoretical calculation supported this interpretation. Photonic crystals infiltrated with liquid crystals and the effect of applied field are also studied and the mechanism of the novel optical property has been clarified.
\end{abstract}

\section{INTRODUCTION}

Photonic crystals, which have a three-dimensionally ordered structure with a periodicity of optical wavelength, have attracted much attention from both fundamental scientific and practical industrial view points, because novel concepts such as photonic band gap and various new types of applications have been proposed [1-2]. As examples we prepared synthetic opals by the sedimentation of silica spheres dispersed in water and also their polymer inverse opals. These opals have interconnected nanosize voids. We demonstrated that various materials can be infiltrated in these void [3-6]. After infiltration of polymer in these opals and then removing silica by washing with HF, polymer inverse opal can be prepared, which has also interconnected regular array of voids. We have proposed tunable photonic crystal crystals utilizing opals and inverse opals infiltrated with various functional materials and demonstrated several examples of tunable photonic crystals [7-10]. In this paper, effects of infiltration of various liquids and liquid crystals in opals and inverse opals are studied and the application of these effects for the evaluation of refractive index of liquid and also for the sensors of liquids and liquid compositions are proposed.

\section{EXPERIMENTAL}

Three-dimensional periodic structures in thin films were fabricated by the sedimentation of mono-dispersed $\mathrm{SiO}_{2}$ spheres of several hundreds $\mathrm{nm}$ in diameter in thin cells. These thin three-dimensional periodic structures of $\mathrm{SiO}_{2}$ (synthetic opals) were heat treated to make them robust. The opal films were confirmed to have a face-centered cubic structure and have interconnected periodic array of voids. The structure was observed by a canning electron microscope (Hitachi, S-2100). The transmission spectrum and reflection spectrum was measured using a spectrophotometer (Hitachi 330).

\section{RESULTS AND DISCUSSION}

Figure 1 shows transmission spectra of an opal and opals infiltrated with various liquids. As evident in this figure, we can observe a sharp dip at some wavelength in the transmission

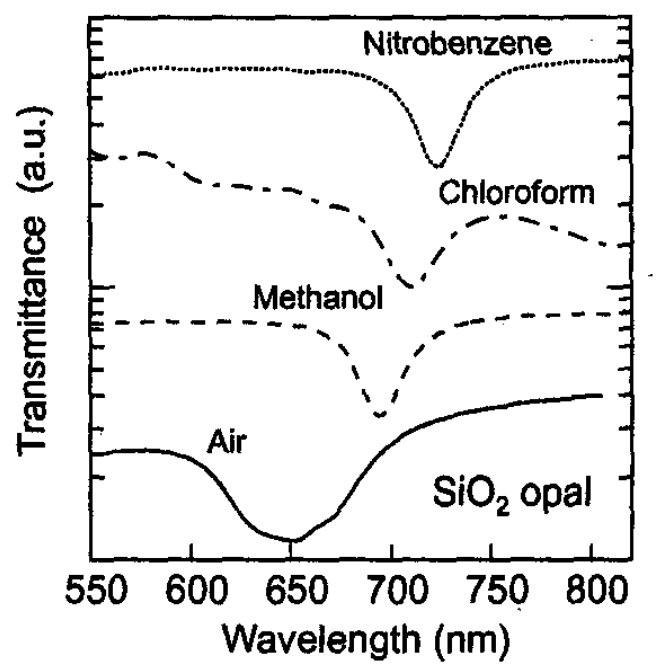

Fig. 1. Transmission spectra of $\mathrm{SiO}_{2}$ opal infiltrated with various liquids. 


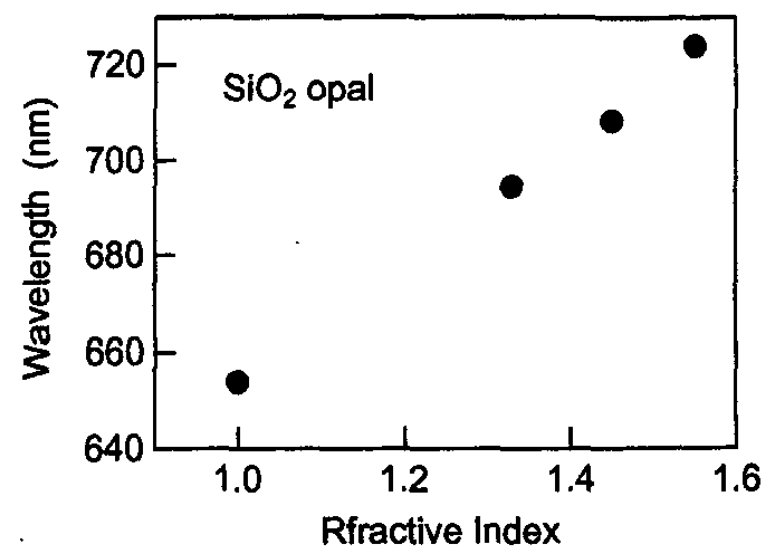

Fig. 2. Transmission dip wavelength of liquid-infiltrated $\mathrm{SiO}_{2}$ opal as a function of refractive index of liquid.

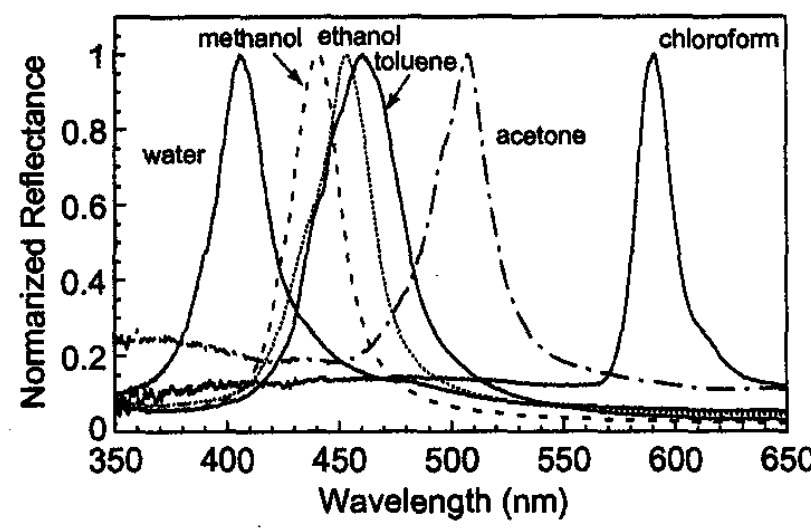

Fig. 3. Reflection spectra of polymer inverse opal infiltrated with various liquids.

spectra, which depends on the sort of liquids. A sharp peak was also observed in a reflection spectrum of the infiltrated opals, which also shifted upon the change of liquids. These spectral shifts of the peak and the dip in the transmission and the reflection spectra can be explained by the difference in the refractive index of the liquids infiltrated in opal.

For normal incidence geometry, Bragg reflection wavelength $\lambda$ is determined using the following equation,

$$
\lambda=2 d \sqrt{f n_{s}^{2}+(1-f) n_{v}^{2}}
$$

where $d$ is the spacing of the (111) planes, which is normal to

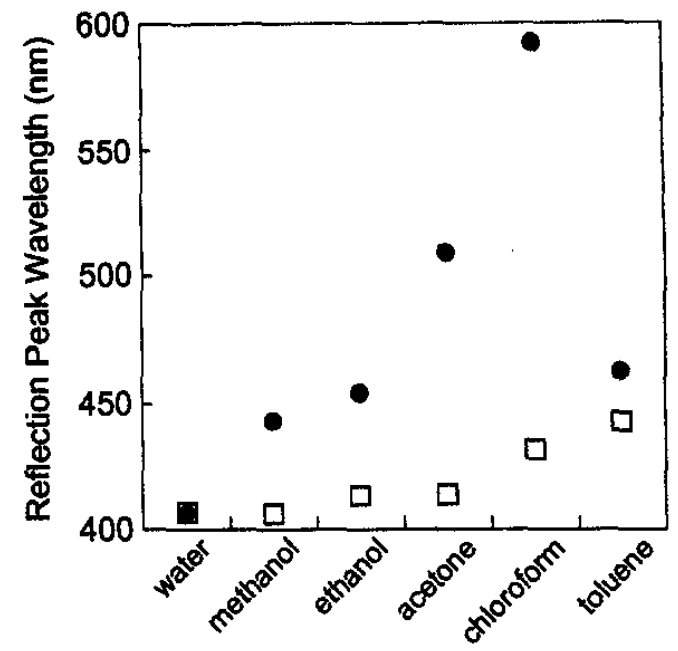

Fig. 4. Reflection peak wavelength of polymer inverse opal infiltrated with various solvents. Closed circles indicate experimental results. Open squares shows wavelength theoretically evaluated assuming constant periodicity.

the light propagation direction, $f$ is the filling factor of the fcc close-packed structure, and $n_{\mathrm{s}}$ and $n_{\mathrm{v}}$ are refractive indices of the $\mathrm{SiO}_{2}$ spheres and substances in the voids of opal, respectively. In the case of non-infiltrated opal, $n_{v}$ is the refractive index of air. When liquids is infiltrated in opal, $n_{\mathrm{v}}$ is for liquids. In this case, $f=0.74, n_{8}=1.46$.

Figure 2 shows the dependence of the dip wavelengths on the refractive index of the solvent. The results are well coincident with the theoretical dependence. That is, the dip wavelength shifts as following the equation (1).

Similar spectral shifts were also observed upon infiltration of liquids in polymer inverse opal as shown in Fig.3. This shift can be explained also by the difference of refractive index of the liquids. However, the shift in the infiltrated polymer inverse opals was much bigger than the case of infiltrated opals, which can be explained in terms of the much larger volume of voids and therefore much larger occupation ratio of liquids in polymer inverse opals compared with the case of opals. In a case of the inverse opal with gel polymer, the shift of the peak and dip was much larger than the value theoretically evaluated utilizing refractive index of the liquids as shown in Fig.4. This anomaly can be explained by the gel characteristics, that is, by the change in volume, therefore the change in periodicity with the liquids. Namely, in this case, changes in not only refractive index but also periodicity must be taken into consideration. It should also be mentioned that graphite inverse opals also exhibited drastic color change with changing solvent. 


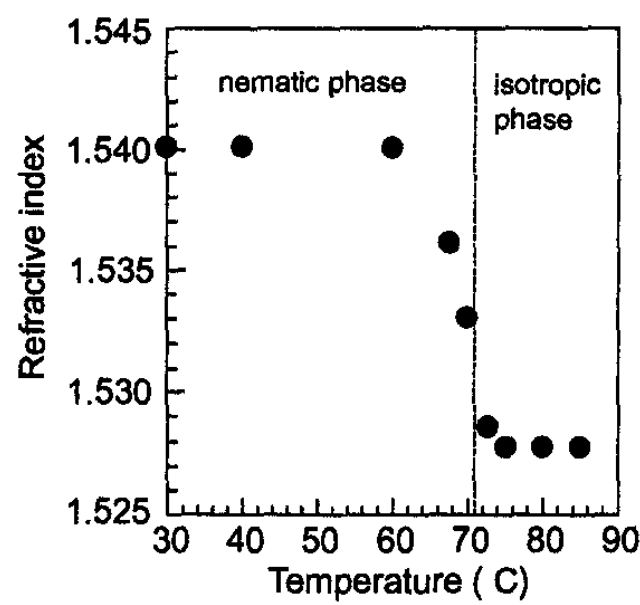

Fig. 5. Temperature dependence of reflection peak wavelength of 5CB-infiltrated opal.

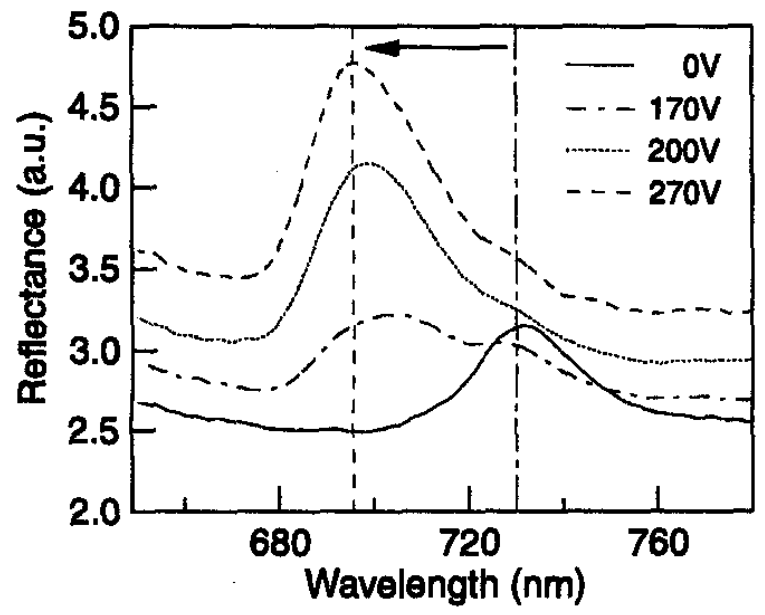

Fig. 6. Reflection spectra of 5CB-infiltrated polymer inverse opal as a function of applied voltage.

Photonic crystals infiltrated with various liquid crystals such as nematic and smectic liquid crystals have also been explored. The reflection peak of the opal infiltrated with liquid crystal shifts with the temperature, especially at the phase transition points, which can be explained also by the change of refractive index with temperature, especially step wise change at the phase transition, as shown in Fig.5. This result is also consistent with the theoretical analysis of photonic crystals infiltrated with liquid crystals.

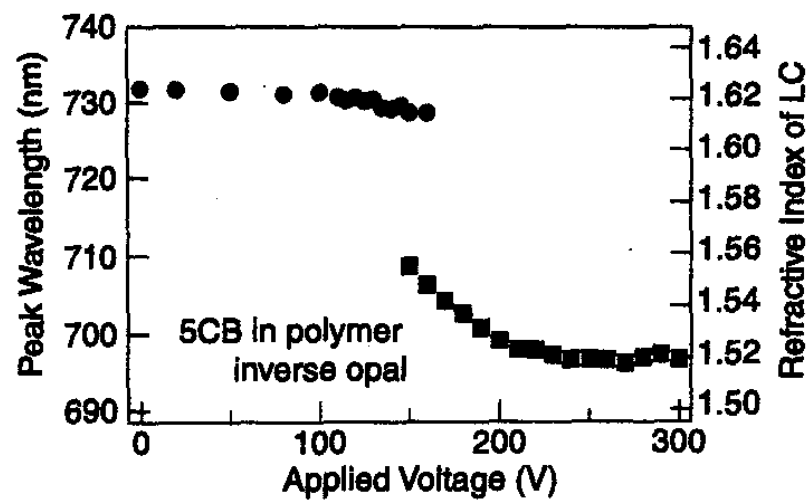

Fig. 7. Voltage dependence of peak wavelength of reflection spectrum of 5CB-infiltrated polymer inverse opal. Refractive index of liquid crystal evaluated from the peak wavelength of reflection spectrum is also shown.

The peak in the reflection spectrum in photonic crystals infiltrated with liquid crystals shifted with applying voltage. Figure 6 shows the reflection spectra of $5 \mathrm{CB}$-infiltrated polymer inverse opal as a function of applied voltage. 5CB (4-pentyl-4'-cyanobiphenyl) is a quite common nematic liquid crystal. As evident from the figure, the reflection peak shifts toward shorter wavelength upon applying voltage. The dependence of the peak wavelength on the applied voltage is more clearly indicated in Fig.7. This shift can be explained by the change of refractive index accompanying with the reorientation orientation of nematic liquid crystals with the applied voltage. The existence of the threshold and the hysteresis due to the anchoring force between the wall of voids and the liquid crystal molecules. The response speed of this voltage induced spectral shift was much faster than the same liquid crystal in a conventional liquid crystal cell.

\section{SUMMARY}

We prepared synthetic opals by the sedimentation of silica spheres dispersed in water, and also fabricated polymer inverse opals from the silica opal. Upon infiltration of various liquids in the opal and inverse opal, a sharp peak in the reflection spectrum and a dip in the transmission spectrum shifted drastically. The wavelength shift strongly depended on the sort of infiltrated liquids. For the polymer inverse opal, larger wavelength shift compared with that theoretically predicted was confirmed, which was interpreted in terms of the volume change in the polymer gel matrix. The optical stop band in the transmission and reflection spectra in the opal and inverse opal infiltrated with liquid crystal could be tuned upon the temperature change or upon the electric field application. 


\section{REFERENCES}

[1] Yablonovitch E. "Inhibited Spontaneous Emission in SolidState Physics and Electronics", Phys. Rev. Lett., Vol.58, No.20, 18 May, 1987, pp.2059-2062.

[2] John S. "Strong localization of photons in certain disordered dielectric superlattices", Phys. Rev. Lett., Vol.58, No.23, 8 June, 1987, pp.2486-2489.

[3] Yoshino K., Tada K., Ozaki M., Zakhidov A.A. and Baughman R.H. "The Optical Properties of Porous Opal Crystals Infiltrated with Organic Molecules", Jpn. J. Appl. Phys., Vol.36, No.6A, 1 June, 1997, pp.L714-L717.

[4] Yoshino K., Lee S.B., Tatsuhara S., Kawagishi Y., Ozaki M. and Zakhidov A.A. "Observation of Inhibited Spontaneous Emission and Stimulated Emission of Rhodamine $6 \mathrm{G}$ in Polymer Replica of Synthetic Opal", Appl. Phys. Lett., Vol.73, No.24, 14 Dec., 1998, pp.3506-3508.

[5] Yoshino K., Tatsuhara S., Kawagishi Y., Ozaki M., Zakhidov A.A. and Vardeny Z.V. "Spectral Narrowing of Photoluminescence in Conducting polymer and Fluorescent Dyes Infiltrated in Photonic Crystal Synthetic Opal", Jpn. J. Appl. Phys., Vol.37, No.10A, 1 Oct., 1998, pp.L1187-L1189.

[6] Yoshino K., Tatsuhara S., Kawagishi Y., Ozaki M., Zakhidov A.A. and Vardeny Z.V. "Amplified Spontaneous Emission and Lasing in Conducting Polymers and Fluorescent Dyes in Opals as Photonic Crystals", Appl. Phys. Lett., Vol.74, No.18, 3 May, 1999, pp.2590-2592.

[7] Yoshino K., Shimoda Y., Kawagishi Y., Nakayama K. and Ozaki M. "Temperature Tuning of the Stop Band in Transmission Spectra of Liquid-Crystal Infiltrated Synthetic Opal as Tunable Photonic Crystal", Appl. Phys. Lett., Vol.75, No.7, 16 August, 1999, pp.932-934.

[8] Yoshino K., Satoh S., Shimoda Y., Kawagishi Y., Nakayama $\mathrm{K}$. and Ozaki M. "Tunable Optical Stop Band and Reflection Peak in Synthetic Opal Infiltrated with Liquid Crystal and Conducting Polymer as Photonic Crystal", Jpn. J. Appl. Phys., Vol.38, Part 2, No.8B, 15 August, 1999, pp.L961-L963.

[9] Shimoda Y., Ozaki M. and Yoshino K. "Electric Field Tuning of Stop Band in Reflection Spectrum of Synthetic Opal Infiltrated with Nematic Liquid Crystal", Appl. Phys. Lett., Vol.79, No.22, 26 Nov., 2001, pp.3627-3629.

[10]Ozaki M., Shimoda Y., Kasano M. and Yoshino K. "Electric Field Tuning of Stop Band in a Liquid-Crystal-Infiltrated Polymer Inverse Opal", Adv.Mater., Vol.14, No.7, 2002, in press. 